\title{
Interpreting manometric signals for propulsion in the gut
}

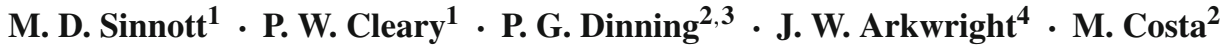

Received: 24 December 2014 / Accepted: 30 May 2015 / Published online: 23 June 2015

(C) Crown Copyright 2015

\begin{abstract}
Propulsion of intestinal contents involves coordinated contractions and relaxations of the muscle controlled by polarised enteric neural reflex pathways. Due to the inaccessibility of the small and large bowel, obtaining detailed manometric measurements in the gut or visualizing the movement of digesta is difficult in vivo. Computational modelling that incorporates the complex interactions between gut content and wall contractility has the potential to explain the mechanisms behind propulsive motor patterns and aid the interpretation of manometric measurements. We present here a biomechanical computational model of coupled wall flexure and flow dynamics in a virtual segment of intestine. The model uses the smoothed particle hydrodynamics method which permits coupling of the fluid/solid motion and wall deformation in a natural way. Peristaltic waves of contraction and relaxation, similar to those observed in physiological experiments, were applied to the gut wall of the model. A catheter containing manometric sensors was also incorporated into the model to derive representative pressure readings. The sensitivity of the model to input parameters including wall stiffness, viscosity of content and degree of muscular contraction is also presented. The results show that there is a rapid rise in pressure of fluid content trapped between the catheter and the contracting wall. The peristaltic
\end{abstract}

M. D. Sinnott

matthew.sinnott@csiro.au

CSIRO Computational Informatics, Clayton, VIC, Australia

2 Department of Human Physiology \& Centre for Neuroscience, Flinders University, Adelaide, SA, Australia

3 Departments of Gastroenterology and Surgery, Flinders Medical Centre, Bedford Park, South Australia, Australia

4 Computer Science, Engineering and Mathematics, Flinders University, Bedford Park, South Australia, Australia wave travels along the length of the virtual segment of intestine passing over each sensor. The bolus, formed by the peristaltic contraction, grows in size and longitudinal extent until the bolus size reaches steady state. The wall force and the peak fluid pressure both scale proportionally with the change in muscle length, indicating that manometric data provide a reliable means for measuring the strength of contractions. Changes in stiffness of the wall and viscosity of contents result in predictable changes in the parameters of peristalsis. The model can be thus applied to manometry measurements in real experimental conditions.

Keywords Peristalsis - Intestinal manometry - Fluidstructure interaction $\cdot$ Smoothed particle hydrodynamics

\section{Introduction}

Progression of intestinal contents relies on coordinated neuromuscular function of the gut. Propulsive movements involve coordinated contractions and relaxations of the circular muscle layer along the gut. The simple hypothesis put forward by Bayliss and Starling [1] suggested that at every point along the gut there are polarised, ascending excitatory and descending inhibitory reflex pathways. A bolus would activate them and thus initiate a sequential activation of the reflexes by the advancing bolus. It took almost 100 years to describe the cellular nature of these enteric neural pathways [2]. Costa et al. [3,4] have developed a method for indirect characterization of circular muscle state during peristalsis in animal models. This method is based on simultaneous manometric measurement and visualization of spatiotemporal variations in wall diameter. Using this method on isolated segments of rabbit colon direct evidence was provided that propulsion of an advancing bolus is associated with activation 
of enteric excitatory motor neurons leading to contraction of the circular muscle towards the oral end and in parallel with activation of enteric inhibitory neurons.

However similar direct studies in vivo of the state of the muscle, with ongoing myogenic and neurogenic processes, are difficult due to lack of visualization of the movement of digesta with the time-varying shape of the lumen. Instead, manometric sensors are often used to investigate motility in vivo $[5,6]$. However such sensors cannot differentiate between intraluminal pressures and the force of the lumen contacting the sensor.

In order to be able to correctly interpret intraluminal pressure signals it would be advantageous to have a suitable biomechanical model of a conventional segment of intestine undergoing mechanical events similar to those observed during propulsive movements, while simulating the intraluminal pressures recorded by the manometry probes. Computational modelling of coupled wall/content dynamics may potentially aid the interpretation of such measurements and provides a means to bridge the gap between animal models and clinical assessment. Few models to date have attempted to adequately treat fluid-structure interactions between flexible wall and viscous fluid. Furthermore, such models also need to be able to include the dynamic local tightening and relaxing of the wall during peristalsis due to changing muscle states $[3,4]$. Detailed computational models of oesophageal motility [7] have helped to define relationships between motility and flow. In addition some models for flow in other parts of the gut [8,9] have used anatomically realistic geometries by completely specifying the wall kinematics and solve for the fluid dynamics alone. This one-way coupling leads to non-physical forces applied to the fluid content with no ability of the rigid wall to respond to local changes in fluid pressure. In this study we have used a biomechanical computational model, previously developed for coupled wall flexure and flow dynamics [10] based on the smoothed particle hydrodynamics (SPH) method [11], to interpret how manometric sensor readings relate to intraluminal pressures in a dynamic model.

\section{Methodology}

\subsection{Simulation methodology}

In the present study we have used the SPH wall flexure model applied to a virtual isolated intestinal segment in which we have simulated a propagating ring of circular muscle, similar to intestinal peristaltic contractions. In addition we have added a virtual manometery catheter to record intraluminal pressures. Thus the model simulated the flow and pressure field that results from a single peristaltic wave traversing the segment. For this, we only considered the state of activation of the circular muscle. Peristaltic travelling waves were simulated on the basis of coordinated activation of polarised enteric neural pathways consisting of a ring of circular muscle contraction advancing in the aboral direction which is preceded by a region of active relaxation [3]. We simulated the effect of this propagating event on the signals of individual manometric sensors spaced along the lumen which represent the manometric probe. The model takes into account changes in muscle fibre length generated by active contractions/relaxations while also being dependent upon the passive compliance of the gut wall and the viscosity of luminal content. A virtual segment of intestine of $6 \mathrm{~mm}$ diameter and $13 \mathrm{~cm}$ long was used as the model segment of intestine.

A brief description of the simulation scheme for each computation timestep is as follows:

1. The instantaneous fluid flow is calculated based on SPH evaluations of the local fluid forces as well as boundary forces arising from the fluid dynamics and the changing shape of the wall.

2. Fluid forces are applied to each boundary node and are summed with the tensile forces in the network of springs representing the wall compliance.

3. The effect of the peristaltic wave is controlled by managing the natural lengths of the wall springs to reflect the current local state of contraction or relaxation.

4. Wall node positions are each calculated by integrating Newton's equations with net force given by the sum of the local fluid and visco-elastic wall forces. This gives an updated shape for the wall geometry.

5. Fluid stresses exerted on the "model sensors" in the catheter are collected and recorded.

\subsection{Fluid model for intraluminal content}

The fluid content is assumed to be Newtonian and modelled using the meshfree numerical method SPH. This method was developed by Monaghan [11] for astrophysical models of large gravitating systems. The details of the CSIRO SPH solver used for this study are well described in $[10,12]$. Briefly, computations are made onto SPH fluid nodes or "particles". These particles move in response to fluid stresses imparted to them and carry details of their local state with them. The adaptive nature of SPH allows for large deformations to be modelled without the overheads of expensive and diffusive re-meshing required for grid based methods.

The SPH continuity equation gives the evolution of the density $\rho_{\mathrm{a}}$ of particle a, as:

$\frac{d \rho_{a}}{d t}=\sum_{b} m_{b}\left(\mathbf{v}_{a}-\mathbf{v}_{b}\right) \cdot \nabla W_{a b}$

where $v_{a}$ is the velocity and $m_{b}$ is the mass of particle $b$. The position vector between particles $\mathrm{b}$ and $\mathrm{a}$ is denoted $\mathrm{as} \mathrm{r}_{\mathrm{ab}}=$ 
Fig. 1 Flexible wall model showing a contraction and the expansion of the wall inside the bolus region. The wall is represented by a network of springs and dashpots
Mesh of 1D bonds (springs/dashpots)

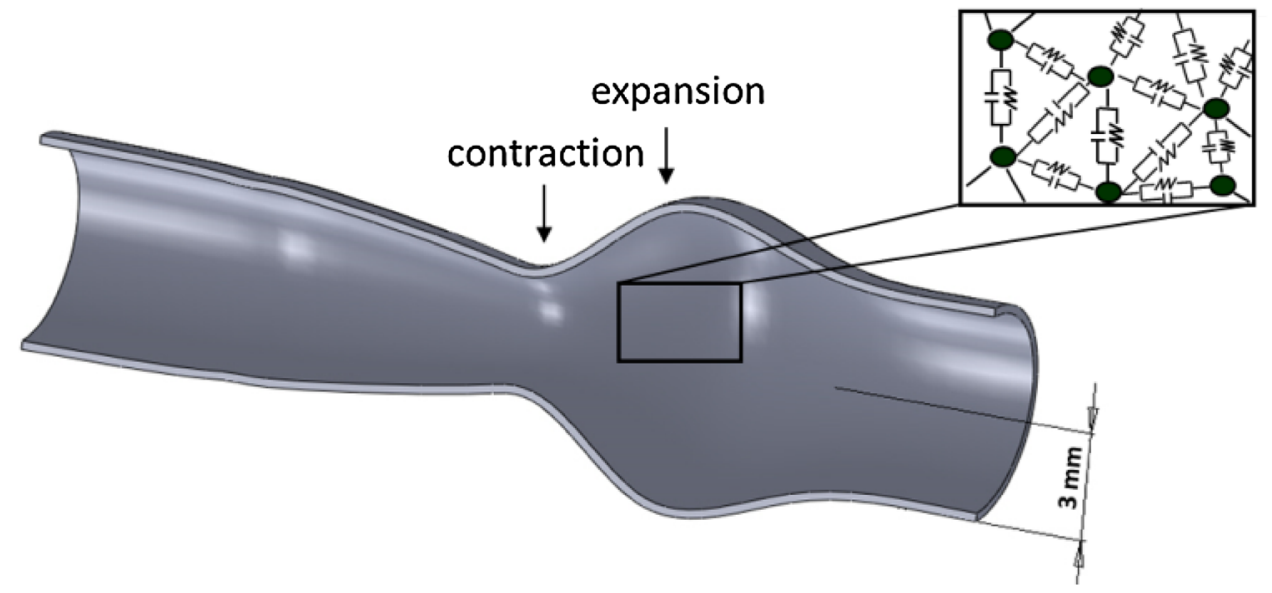

$r_{a}-r_{b}$. The value of the interpolation kernel with smoothing length $\mathrm{h}$ evaluated for the distance $\left|\mathrm{r}_{\mathrm{ab}}\right|$ is $\mathrm{W}_{\mathrm{ab}}=\mathrm{W}\left(\mathrm{r}_{\mathrm{ab}}, \mathrm{h}\right)$. This form of the continuity equation is very effective for modeling free surfaces and has good numerical conservation properties.

The momentum equation for a Newtonian fluid with strongly variable viscosity is:

$$
\begin{aligned}
& \frac{d \mathbf{v}_{a}}{d t}=\mathbf{g}-\sum_{b} m_{b} \\
& \quad\left[\left(\frac{P_{b}}{\rho_{b}^{2}}+\frac{P_{a}}{\rho_{a}^{2}}\right)-\frac{\xi}{\rho_{a} \rho_{b}} \frac{4 \mu_{a} \mu_{b}}{\left(\mu_{a}+\mu_{b}\right)} \frac{\mathbf{v}_{a b} \mathbf{r}_{a b}}{\mathbf{r}_{a b}^{2}+\eta^{2}}\right] \nabla_{a} W_{a b}
\end{aligned}
$$

where $P_{a}$ and $\mu_{a}$ are the pressure and fluid viscosity for particle a. The velocity difference between particles is $\mathrm{v}_{\mathrm{ab}}=$ $v_{a}-v_{b}$ and $g$ is the gravity vector. Here $\eta$ is a small parameter used to smooth out the singularity at $r_{a b}=0$ and $\xi$ is a factor associated with the viscous term. This formulation gives good momentum conservation in the presence of very high spatial viscosity gradients.

A quasi-compressible approach is used for linking the pressure and density of each particle using an equation of state:

$P=P_{0}\left[\left(\frac{\rho}{\rho_{0}}\right)^{\gamma}-1\right]$.

Here $\mathrm{P}_{0}$ is a problem specific pressure scale and $\rho_{0}$ is a reference density. We use $\gamma=7$ corresponding to the behavior of incompressible liquids and then use an appropriate speed of sound so as to model the fluid flow near the incompressible limit.
Rigid boundaries such as the manometric catheter may be included where the fluid-boundary interaction makes use of Lennard-Jones forces. The mesh-free nature of SPH permits the straightforward treatment of multiphase flows so that coupled wall flexure and fluid flow are captured naturally. Biomechanical models based on this method have been developed for cardiovascular flow [13], biomedical devices [14] and sports applications involving full body motions [15].

\subsection{Flexible wall model}

The flexible wall model has been well described in [10]. Biological soft tissues are visco-elastic and often anisotropic in nature [16]. The real gut wall is comprised of multiple layers of soft tissue each with different material properties. In particular, circular smooth muscle in the gastrointestinal tract has been shown to be highly visco-elastic [17]. The animal experiments that this study represents constrain the muscular activity to circular muscle only. This simplifies the system and therefore we make the choice to represent the gut wall as a single, isotropic membrane. This includes passive compliance of the wall so that the membrane generates tensile forces if stretched beyond its natural length in any direction. For this study, the section of intestine is a cylindrical geometry with closed ends and is initially filled with just enough fluid so that there are no void spaces and therefore no tensile forces generated in the walls when at rest. The inner surface of the gut wall shown in Fig. 1 is represented by a mesh of triangular elements where the nodes of the mesh are SPH fluid particles. Interactions between the fluid and wall are then treated naturally via fluid forces using the SPH method. Compliance of the gut wall is modelled using the node connectivities as a network of visco-elastic springs. This is a coarse approximation of the behaviour of muscle fibres assuming that muscle force is proportional to change in fibre extension from rest 
state. The elastic phase is represented by a series of Hookian elements (springs) and the viscous phase by Newton elements (dashpots) which controls the rate at which the wall can extend or contract. The elastic compliance of the wall is therefore fully defined by a stiffness parameter related to the elasticity of the muscle fibres and an equilibrium rest length related to the rest state of the fibres. This model allows for elastic deformation of the lumen in response to internal content pressures or muscle activation due to the passage of a peristaltic wave. The instantaneous shape of the wall is then a direct prediction from the fluid-structure interaction between intra-luminal content and muscular control of the boundary.

\subsection{Simulation of peristaltic waves}

In our simulation we modeled a local activation of circular muscle. The rest lengths of the elastic wall elements in each longitudinal slice of lumen were then controlled by the passage of the wave. These elements (representing muscle fibres) in the virtual gut wall in addition to responding to passive forces will shorten by an active contraction and lengthen by an active relaxation. As far as the authors know, the shape of the peristaltic waveform is not known. For each wave we maintained smooth variation with distance by describing each contraction (or relaxation) by a cosine function so that the peak of the contraction (relaxation) occurs at the centre of the activated region and falls to zero outside this region.

\subsection{Representation of manometric sensors in the model}

For the flow simulations in this study, individual model pressure sensors were included based on high-resolution fibre-optic manometry developed by Arkwright et al. [18]. The model catheter contained 12 sensors located at $\mathrm{z}=$ $0.5+\mathrm{n}$ cm intervals where $\mathrm{n}=(0,1,2, \ldots, 11)$. The design of the fiber-optic catheter meant that adjacent sensors faced in opposite directions (i.e. alternating between 12 o'clock and 6 o'clock). The virtual fibre-optic manometry catheter incorporated these characteristics.

The catheter and individual sensors were represented in the simulations by a surface mesh of triangular elements. The total fluid force exerted on each element is recorded every $0.007 \mathrm{~s}$ during the simulation and assigned to its parent sensor. The total force for each sensor is then converted to manometric pressure and reported as a pressure time series.

\subsection{Simulation strategy}

Simulations were performed with the coupled flexible wall/ flow model to investigate the dependence of material parameters for the wall and content on the intraluminal pressure field and pressure sensor readings. A base case simulation was performed with a $40 \%$ contraction, a wall stiffness of
Table 1 Simulation conditions used in this study

\begin{tabular}{llll}
\hline Label & $\begin{array}{l}\text { Wall stiffness } \\
(\mathrm{Nm})\end{array}$ & $\begin{array}{l}\text { Occlusion } \\
(\%)\end{array}$ & Viscosity (Pas) \\
\hline Base case & 10 & 40 & 0.001 \\
Stiffness1 & 20 & 40 & 0.001 \\
Stiffness2 & 50 & 40 & 0.001 \\
$60 \%$ Occ & 10 & 60 & 0.001 \\
$80 \%$ Occ & 10 & 80 & 0.001 \\
Visc1 & 10 & 80 & 0.003 \\
Visc2 & 10 & 80 & 0.01 \\
\hline
\end{tabular}

$10 \mathrm{Nm}$ and a content viscosity of 0.001 Pa s. This simulation highlights the major flow features for the coupled SPH-wall flexure model and provides a reference for comparing other model variants. Different scenarios were simulated for this system varying the strength of contraction $(40,60,80 \%)$, wall stiffness/compliance $(10,20,50 \mathrm{Nm})$, and viscosity of content $(0.001,0.003,0.01 \mathrm{Pas})$. The simulation parameters being varied for each case are summarised in Table 1. A single peristaltic contraction was simulated with active contractile and active relaxation components both commencing a third of the way along the virtual segment and traveling the remaining length of the segment aborally at $1 \mathrm{~cm} / \mathrm{s}$. Pressure readings were spatially integrated onto the model sensors at each time.

\section{Results and discussion}

\subsection{Simulation of peristaltic contraction in the virtual intestine}

\subsubsection{Flow and pressure fields for the base case}

Simulated example of a peristaltic contraction is shown in Fig. 2. The images show the onset of a peristaltic wave and the changes in fluid pressures and flow speed, along an axis directed from oral to anal, as the wave of the propagating contraction develops. In the contraction region, the wall moves radially inward towards the catheter. This occurs within a timescale defined by the viscous phase of the wall elements and the resistance of the fluid content to move sideways out of the way. Pressure begins to rise immediately in front of the contraction and (to relieve this pressure) fluid moves sideways. Relaxation of the wall in the relaxed region accommodates this sideways fluid displacement and the wall progressively dilates in the radial and longitudinal directions to form a bolus shaped content. The expansion of the wall in the relaxed region reduces downstream pressure and leads to greater thrust so that, near the centre of the gut, content 


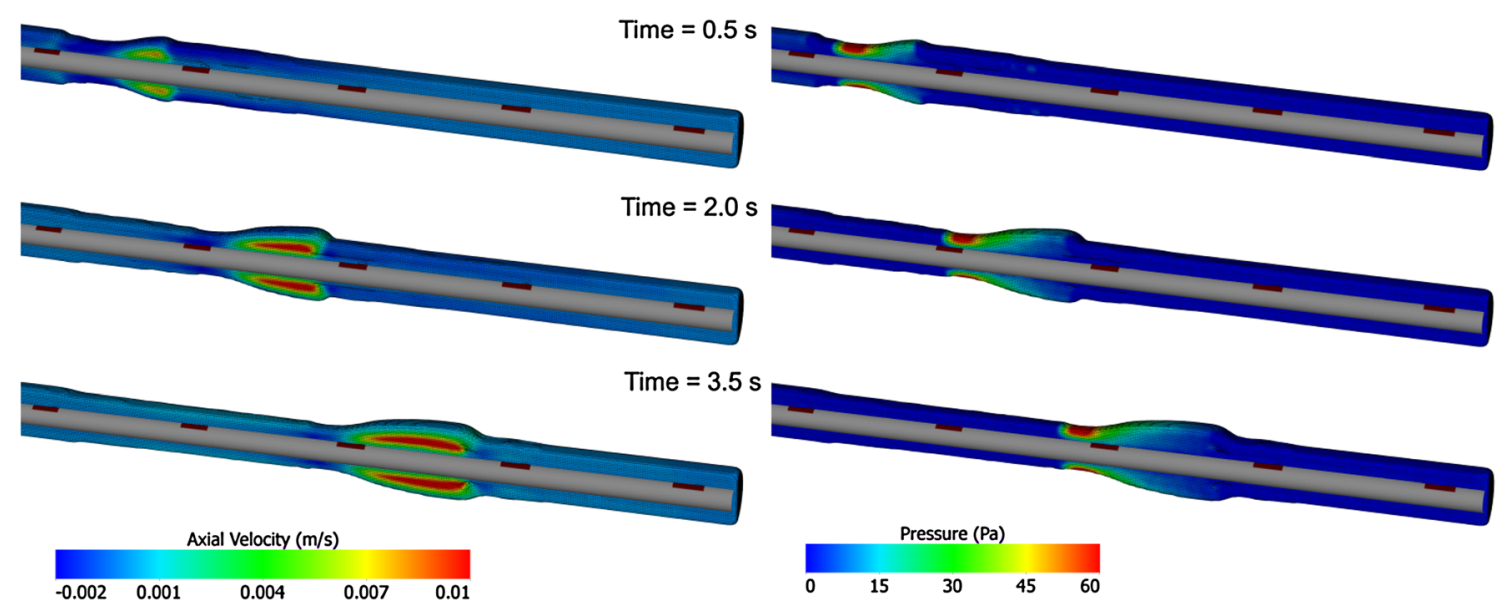

Fig. 2 The development of a single peristaltic wave in the segment of rabbit gut with content coloured by axial speed (to the left) and fluid pressure (to the right). The model sensors are coloured brown and have alternating positions above and below the catheter

is propelled forward at the speed of the propagating contraction. The flow of content is slowed by the presence of the non-moving catheter and also near the gut wall. The variation in axial speed with radial distance from the centre of the gut produces a toroidal recirculation vortex. This recirculation is responsible for driving a high radial pressure gradient that dilates the wall inside the relaxed region, giving the bolus its characteristic elliptical shape. As the wave develops, the vortex ring and bolus grow in size and longitudinal extent until the bolus size reaches steady state. The pressure field shows that the high radial pressure gradient develops almost immediately and is present throughout the growth of the bolus.

\subsubsection{Interpretation of manometric readings}

Pressure data for the intraluminal content were collected by simulated sensors. Figure 3 shows virtual sensor readings from the peristalsis simulation for sensors 8 through 13. This shows the full aboral passage of a single peristaltic wave described in the previous section. The pressure profile for sensor 8 contains the imposed onset of the contraction. The initial steep leading edge is due to the rapid rise in pressure of fluid content trapped between the catheter and the contracting wall. The peristaltic wave travels along the length of the virtual segment of intestine passing over each sensor. For sensors 9-13, the leading edge of the profile shows the gentle gradient of fluid pressure into the formed bolus ahead of the contraction.

As shown in Fig. 2, the fluid bolus grows in size until the build-up of fluid in front of the contraction fully dilates the actively relaxed region. From sensor 10-13, the pressure profile appears steady. The trailing edge falls off rapidly due to a loss of fluid and therefore fluid pressure in the region where the wall makes contact with the sensor.

\subsection{Sensitivity to material properties of the lumen}

\subsubsection{Degree of muscle shortening}

Active contraction of muscle fibres in a single ring of circular muscle results in radial contraction of the lumen wall at a single location. Similarly dilation of the wall occurs in regions of active relaxation, where the muscle fibres increase in length. The flexible wall model incorporates such fractional changes in muscle fibre length by modifying the resting lengths of the wall elements in the network in the circumferential direction, thus resulting in radial contraction. Simple visual inspection of the wall diameter for individual peristaltic events cannot identify the actual changes in muscle fibre length or the tensile forces in the gut wall. This is because the wall shape next to the contraction is due to the balance between tensile forces in the wall and pressure of the fluid content. Therefore it is not currently possible to determine a priori the strength of real contractions and relaxations to include in the simulations. It may however be possible to infer these by comparing simulation with manometry for a given event. Note, in all cases the central rigid catheter is intended to impose a full occlusion condition (even for contractions that would otherwise only partially occlude the lumen). This enables us to regard "isometric contractions" where the wall tension changes but the wall diameter does not.

Figure 4 shows the simulated response of the intraluminal pressure field to different strength contractions, corresponding to fractional shortening of muscle fibre lengths by 40,60 and $80 \%$ of the resting length. Pressures in the contraction region rise significantly more for stronger contractions. The pressure on the catheter also increases proportionally with strength of the contraction. Wall diameters in the contraction region are constant over a larger region for the $80 \%$ case since more of the surrounding muscle is now included in the 

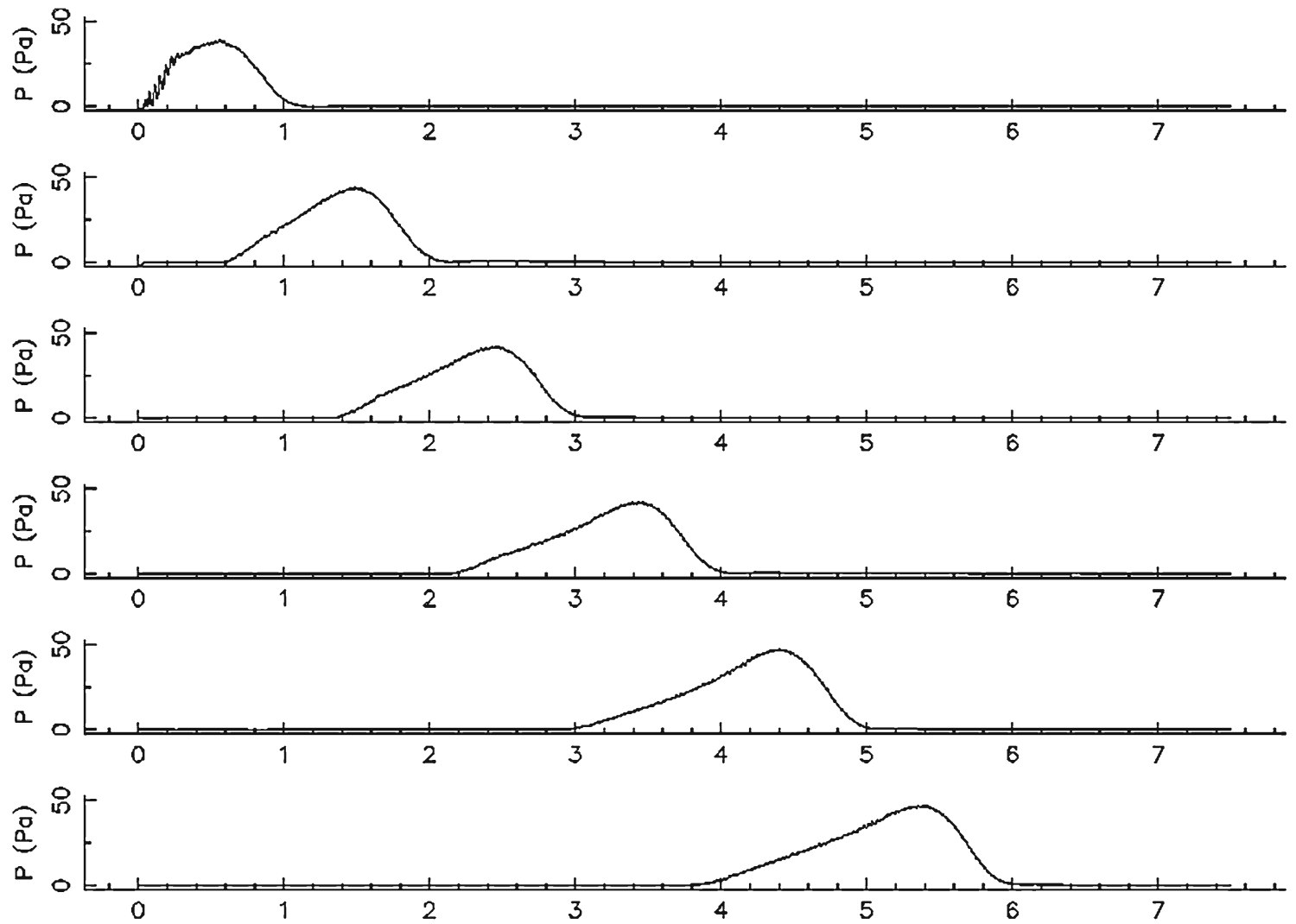

Fig. 3 Simulated manometric sensor readings demonstrating transit of peristaltic wave in the wall corresponding to transport of the fluid bolus along the length of gut segment
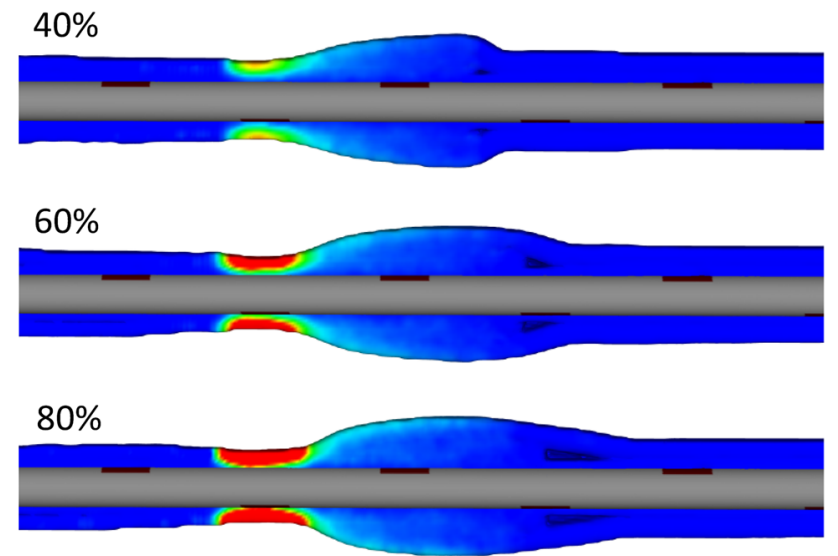

Pressure (Pa)

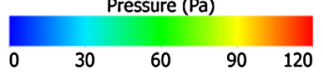

Fig. 4 Effect of fractional shortening of circular muscle on details of the flow in the contraction/relaxation region

isometric contraction. The shape of the bolus changes from a teardrop at $40 \%$, to a symmetric ellipse at $60 \%$ and finally to a reverse teardrop shape at $80 \%$. Despite the change in shape, the intraluminal pressure distribution inside the bolus

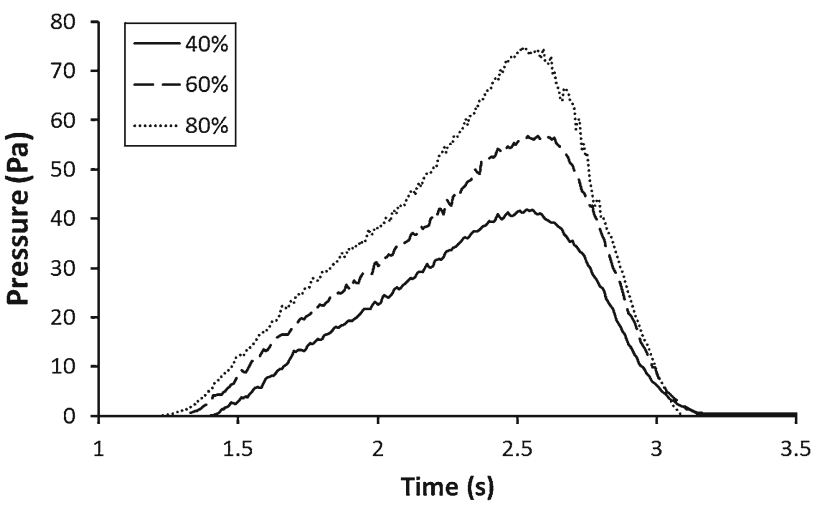

Fig. 5 Manometric pressure profiles measured at sensor 10 for different fractional shortening of circular muscle $(40,60$ and $80 \%)$

remains very similar in the three conditions. This leads to very similar pressure profile shapes for the sensor readings shown in Fig. 5.

The change in peak fluid pressure (recorded by the model sensor) scales roughly proportionally with the fractional reduction in muscle fibre length. This is reasonable since the tensile force in the wall is proportional to the extension of the elastic wall elements. The leading edge is similar in gradient for each contraction indicating that the pressure dis- 

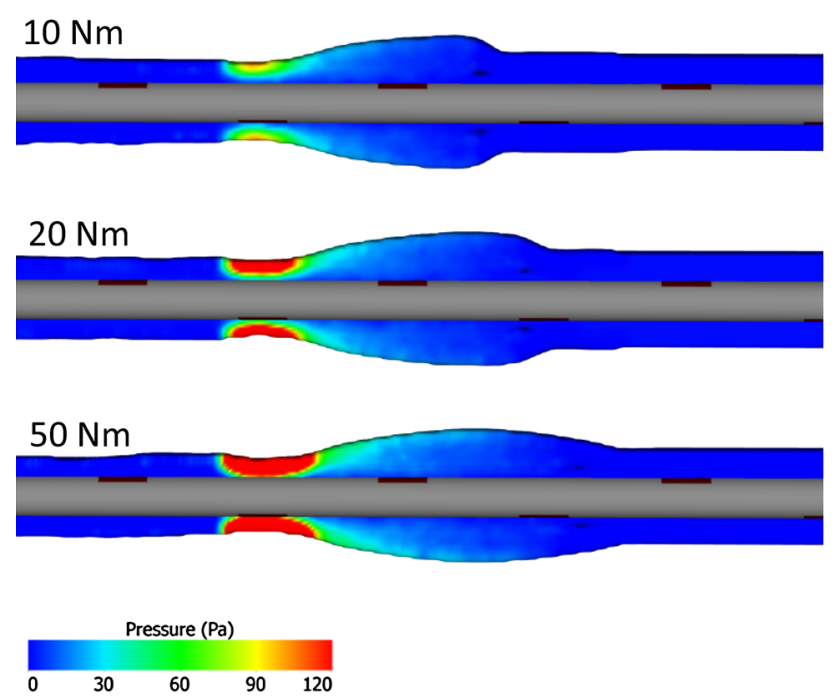

Fig. 6 Effect of wall stiffness on pressure field inside the contraction/relaxation region

tribution inside the bolus region is very similar for the three cases. The shape of the trailing edge also appears insensitive to the increase in wall tension. This suggests that the downstream contact point between wall and sensor does not depend on the strength of an isometric contraction. The wall force (from Fig. 4) and the peak fluid pressure (from Fig. 5) both scale proportionally with the change in muscle fibre length. Therefore manometric data for a single isometric contraction should provide a reliable and direct means for measuring the strength of the contraction.

\subsubsection{Wall stiffness}

For accurate simulation of the dynamic response of the wall to intraluminal pressures, knowledge of the intrinsic material properties is required. However, direct measurement of the elastic modulus of relaxed muscle tissue is not straightforward. Measurements of bulk stiffness/compliance of soft tissue, such as intestinal wall must be carried out under static conditions and depend on the load applied to the tissue [16]. As such it is not straightforward to apply these measurements to the rapid dynamic changes during intestinal movements.

The wall stiffness we refer to here is the stiffness value used for all elastic elements in the mesh network representing the intestinal wall. A change in element stiffness modifies the tensile force in the wall for a given element extension and therefore will result in changes in compliance of both passive and active tissue throughout the entire segment. Figure 6 shows the effect of wall stiffness for low $(10 \mathrm{Nm})$, medium $(20 \mathrm{Nm})$ and high $(50 \mathrm{Nm})$ stiffness values on the intraluminal pressure distribution and the wall shape.

There is a proportional rise in wall force at the contraction site with increasing stiffness. This is because the radial force

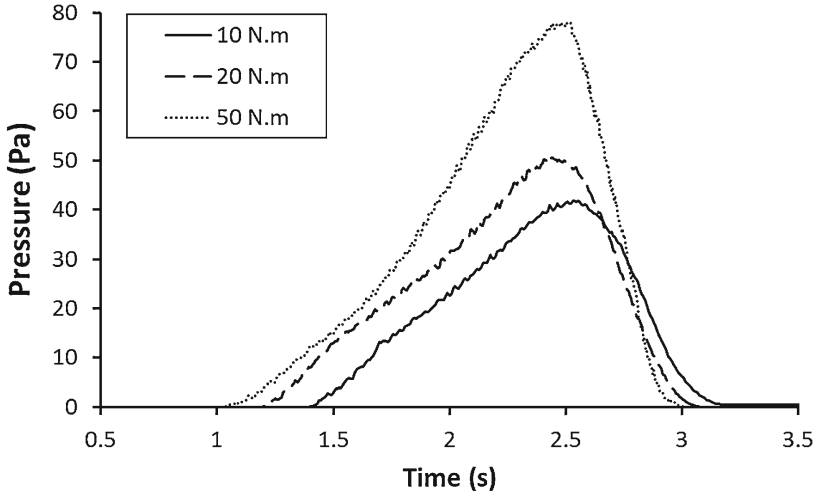

Fig. 7 Manometric pressure profiles measured at sensor 10 for different stiffness $(10,20,50 \mathrm{Nm})$ of the lumen

is proportional to tensile force for isometric contractions. Fluid pressures rise and extend further into the bolus region at higher stiffness. The tensile force in the actively relaxed region, as well as the surrounding passive tissue outside the active peristaltic contraction and relaxation, also increases proportionally with stiffness. This leads to an increase in pressure along the wall of the relaxation region at high stiffness. Together with the increased wall tension, this increase in pressure modifies the size and shape of the bolus. For medium to high stiffness, the bolus appears more elongated and symmetrical in shape in the lengthwise direction. The manometric readings for the fluid pressures in Fig. 7 show that the peak fluid pressure immediately in front of the contraction doubles with a fivefold increase in stiffness whereas the wall force (from Fig. 6) shows a proportional increase. Between the low and high stiffness cases, there is a delay of $0.4 \mathrm{~s}$ in the arrival of the first pressures recorded by the sensor. This is consistent with the higher pressures observed extending further inside the bolus. With increasing stiffness, the trailing edge becomes steeper and the recorded fluid pressure falls to zero earlier. This indicates that at high stiffness there is a small increase in the contact area of the wall pressing onto the catheter. So the first contact point between wall and catheter extends somewhat further downstream.

\subsection{Sensitivity to fluid content properties}

\subsubsection{Effect of fluid viscosity}

The viscosity of luminal content influences how the gut wall responds to intraluminal pressures, and how fluid content responds to wall force. Physiological viscosity of natural contents varies along the intestine, from fluid to semi-solid. This is a consequence of water absorption during content passage along the intestine.

We investigated the effect of varying the viscosity of luminal contents on the pressure field of the content and the 

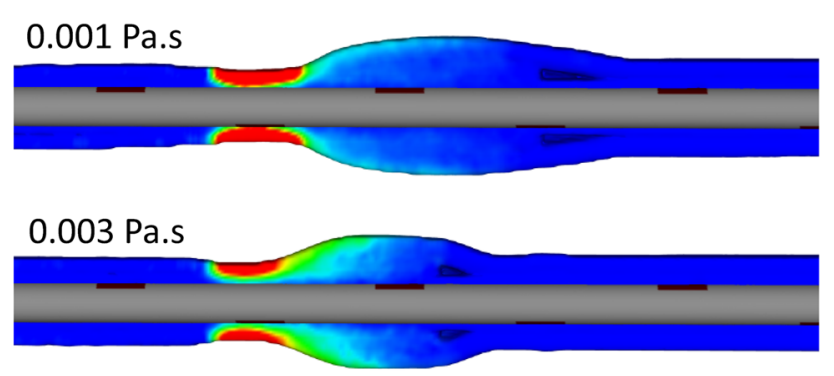

0.01 Pa.s
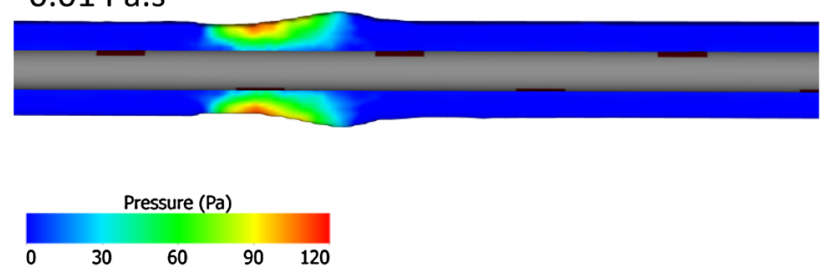

Fig. 8 Effect of viscosity of content on pressure field inside the contraction/relaxation region for an $80 \%$ shortening of muscle fibres

resulting sensor data. Figure 8 shows the pressure field for low (0.001 Pas), medium (0.003 Pas) and high (0.01 Pas) viscosities. These simulations use a contraction with $80 \%$ shortening of muscle fibres in order to significantly perturb the higher viscosity content. At low viscosity, greater fluid mobility enables the generation of a recirculation vortex inside the bolus described above. A threefold increase in viscosity reduces the size of the vortex and therefore the size and shape of the bolus. Fluid pressures inside the bolus rise, most noticeably close to the intestinal wall. Due to the smaller bolus size, the pressures are now confined to a more compact region. Peak wall force at the contraction reduces by approximately half. The higher resistance to shear for content between the contracting wall and catheter means the speed of shortening of the circular muscle slows down. This limits the contact time between wall and catheter and prevents full contraction of the muscle in the time available before the peristaltic wave has moved downstream. Thus, the transmission of force from wall to sensors is reduced. At high viscosity, the contraction is not strong enough to push content out of the way in time before the wave moves downstream. Therefore full occlusion does not occur and there is no direct contact between wall and catheter. The bolus has almost vanished indicating that the presence of the vortex and radial pressure gradient is suppressed at high viscosities. Reduced mobility of content leads to higher fluid pressures inside a compact region around the contraction which moves with the peristaltic wave. The flow changes from radial transport at low viscosity to almost entirely in the lengthwise direction but with reduced efficiency at high viscosity.

Fluid pressures recorded by the model sensor in Fig. 9 show a rise in peak pressure with viscosity even as the wall force decreases. Force transmission from wall to sensor

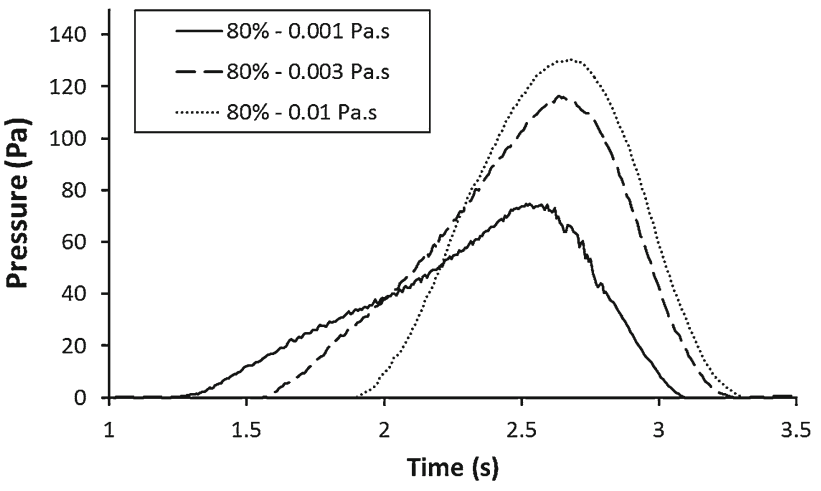

Fig. 9 Manometric pressure profiles measured at sensor 10 for different fluid viscosities $(0.001,0.003,0.01 \mathrm{Pas})$ for an $80 \%$ contraction

changes from direct contact (dominant at low viscosity) to mediation via the fluid content trapped between contracting wall and catheter (at high viscosity). This manifests at the sensor as a delay in the arrival time for the peak fluid pressure. The location of peak pressure shifts from a point just in advance of the contraction (at $0.001 \mathrm{Pas}$ ) to directly underneath the peak of the contraction (at $0.01 \mathrm{Pas}$ ). The leading edge of the pressure profile becomes steeper with increasing viscosity as the bolus size reduces. At high viscosity, the pressure profile is symmetric about the arrival time of the contraction. This suggests that manometric data will tend to be dominated by fluid forces rather than wall force in the presence of highly viscous content.

\section{Conclusions}

The coupled, flexible wall, SPH model that we have used to simulate peristalsis in a fluid-filled segment of virtual segment of intestine, while recording intraluminal pressures by a manometric catheter, provides a new perspective about the relation between intraluminal pressure field and wall force consistent with biomechanical principles of fluid propulsion. In parallel the simulations also give information on the pressure profiles expected by recording intraluminal pressures with a pressure probe that has been used both in human subjects and animal experiments. This approach gives a greater credibility to the interpretations of manometric measurements.

The full development of a peristaltic wave (with propagating rings of active contraction and relaxation) generates a toroidal vortex inside the relaxed region, which gives the fluid bolus its shape. Manometric measurements were incorporated into the simulations via the addition of model sensors. These calculated the total fluid force exerted onto the surface area of a given sensor at each time but not the wall force. Sensor readings were found to be sensitive to specific mater- 
ial properties of the gut wall and the content. Wall force and peak fluid pressure, for isometric contractions, were both found to scale broadly in proportion to the fractional change in muscle length. This confirms that the strength of the contraction (and intraluminal pressures) increase proportionally with the shortening of muscle length. Stiffer gut walls result in greater wall tension thus reducing wall compliance. This causes fluid pressures to increase and extend further into the relaxed region, increasing the size (diameter, shape and length) of the fluid bolus. The peak wall force in the contraction region rises in proportion with a fivefold change in spring stiffness, whereas the peak fluid pressure only doubles. Therefore the intraluminal pressure field, which drives flow of contents, does not vary greatly over the range of stiffness values examined. This suggests that many aspects of the flow and pressure fields in the gut are not sensitive to material stiffness. Viscosity of content influences the response of the gut wall to intraluminal pressures. Mobility of fluid is important for development of the recirculation vortex. Therefore it is strongest at low viscosities but vanishes at higher viscosities. In the latter case, the radial pressure gradient disappears and flow becomes predominantly axial transport. With highly viscous content, the rate of contraction is slowed and there is insufficient time for the wall to directly contact the catheter before the wave passes aborally. The pressures are then transmitted by the contents between the contraction area and the catheter. The peak fluid pressure increases with viscosity and the location of this peak moves to a point directly underneath the contraction.

There are some important model limitations which constrain the scope of where this model may be used to study real, physiological measurements in a clinical environment. This is the ultimate end goal for this research. The gut wall has been represented here as a single, isotropic, visco-elastic membrane whereas the real gut wall contains multiple layers of soft tissue and muscle each with differing material properties and highly directional and anisotropic behaviour. For the purposes of this work, we consider only two effects (which are sufficient for the current work) these being passive elastic compliance of the gut wall and active contraction and relaxation of circular muscle only. Previous comparisons with experiment indicate that the model predictions of the degree of contraction, the shape of the bolus and the relaxation of the walls following the contraction all give reasonable qualitative agreement. This indicates that the current model appears suitable for studies using circular muscle activity. Another limitation is that this model does not treat the multiphase nature of real digesta which can have a large viscosity range, non-Newtonian behaviour and consist of mixtures of solid, liquid and gas. The effect of multiphase content on manometric sensors is likely to be complex and difficult to interpret without an appropriate model to assist. SPH is a method that is suitable for representing spatially varying viscosities and different phases and these features will progressively be incorporated into this model. The current study considered only Newtonian content which is appropriate for these animal experiments. The results from the current model are representative of the current experimental conditions.

This work proposes a model for interpreting manometric recordings. It also represents a powerful computing tool for simulating intestinal motor patterns observed in human subjects and animal experiments and subsequent validation of the model itself when physiological parameters will be introduced.

Acknowledgments The work was performed under an ARC Discovery Grant (DP120102192). Dr. Sinnott was supported in part by this grant. Dr. Cleary and Prof. Arkwright were supported by funding from CSIRO, Australia.

\section{References}

1. Bayliss WM, Starling EH (1899) The movements and innervations of the small intestine. J Physiol 24:99-143

2. Furness JB, Jones C, Nurgali K, Clerc N (2004) Intrinsic primary afferent neurons and nerve circuits within the intestine. Prog Neurobiol 72(2): 143-164

3. Dinning PG, Wiklendt L, Omari T, Arkwright JW, Spencer NJ, Brookes SJ, Costa M (2014) Neural mechanisms of peristalsis in the isolated rabbit colon: a neuromechanical loop hypothesis. Auton Neurosci 8:75

4. Costa M, Wiklendt L, Arkwright JW, Spencer NJ, Omari T, Brookes SJ, Dinning PG (2013) An experimental method to identify neurogenic and myogenic active mechanical states of intestinal motility. Front Syst Neurosci 11:7

5. Dodds WJ (1976) Instrumentation and methods for intraluminal esophageal manometry. Arch Intern Med 136(5):515-523

6. Torsoli A, Arullani P, Casale C (1967) An application of transintestinal intubation to the study of the colon. Gut 8(2):192-194

7. Brasseur JG, Nicosia MA, Pal A, Miller LS (2007) Function of longitudinal vs circular muscle fibers in esophageal peristalsis, deduced with mathematical modelling. World J Gastroenterol 13:1335-46

8. Udaykumar HS, Krishnan S, Dillard S, Marshall JS, Schulze K (2006) Computation of peristaltic transport and mixing in the small intestine. J Biomech 39:S422

9. Ferrua MJ, Singh RP (2010) Modeling the fluid dynamics in a human stomach to gain insight of food digestion. J Food Sci 75:R151-R162

10. Sinnott MD, Cleary PW, Arkwright JW, Dinning PG (2012) Investigating the relationships between peristaltic contraction and fluid transport in the human colon using smoothed particle hydrodynamics. Comput Biol Med 42:492-503

11. Monaghan JJ (2005) Smoothed particle hydrodynamics. Rep Prog Phys 68:1703-1759

12. Cleary PW, Prakash M, Ha J, Stokes N, Scott C (2007) Smooth particle hydrodynamics: status and future potential. Prog CFD 7:70-90

13. Sinnott MD, Cleary PW (2006) An investigation of pulsatile blood flow in a bifurcation artery using a grid-free method. In: Proceedings of the 5th International Conference on CFD in process industries, Melbourne

14. Sinnott MD, Cleary PW (2010) Effect of rotor blade angle and clearance on blood flow through a non-pulsatile, axial, heart pump. Prog CFD 10:300-306 
15. Cleary PW, Cohen RCZ, Sinnott MD, Prakash M, Mead S (2013) Prediction of industrial, biophysical and extreme geophysical flows using particle methods. Eng Comput 30:157-196

16. Gregersen H (2002) Biomechanics of the gastrointestinal tract: new perspectives in motility research and diagnostics. Springer, London

17. Liao D, Sevcencu C, Yoshida K, Gregersen H (2006) Viscoelastic properties of isolated rat colon smooth muscle cells. Cell Biol Int $30: 854-858$
18. Arkwright JW, Dickson A, Maunder SA, Blenman NG, Lim J, O'Grady G, Archer R, Costa M, Spencer NJ, Brookes S, Pullan A, Dinning PG (2013) The effect of luminal content and rate of occlusion on the interpretation of colonic manometry. Neurogastroenterol Motil 25:e52-e59 